\title{
Multi-directional feature positioning retrieval method of random encrypted images
}

\author{
Fuwei Huang \\ School of Computer Science and Technology, Hainan University \\ Haikou, 570228 \\ China
}

Received: February 24, 2021. Revised: July 18, 2021. Accepted: August 6, 2021. Published: August 11, 2021.

\begin{abstract}
Image encryption is an effective means to ensure image information security, but image encryption makes image features hidden, resulting in blurred image positioning features and cannot provide queryable rules. In this paper, the multi-direction feature retrieval method of random encrypted images is studied comprehensively. Multidirectional binary wavelet is used to decompose specific images, and multi-resolution analysis is used to extract multi-direction features of specific images. The method of image location optimization in random encrypted images is used to eliminate the excessive and repeated image features contained in the specific image by image verification, and the probability of image location errors is reduced. The specific image is retrieved by identifying frequent item sets in random encrypted images that are identical to the multidirectional features of a particular image. The results show that the method can locate the random encrypted image effectively. The accuracy of the image location and the average accuracy of the feature points are about $95 \%$ and $97.3 \%$ respectively, and the anti-noise ability is strong. It provides a scientific means for the rapid positioning of efficient images.

Keywords-Features, frequent item set, image verification, multiple directions, positioning retrieval, random encrypted image.
\end{abstract}

\section{INTRODUCTION}

$\mathrm{W}$ ITH the development of information technology, people pay more and more attention to the importance of image security. In order to prevent image information leakage, improve the security of image information, it is necessary to encrypt images [1]. Because image encryption makes the image feature hidden, the image positioning feature is blurred, and it cannot provide queryable rules [2]. Therefore, it is necessary to study the location retrieval method of specific images in random encrypted images.

There have been many studies on the method of specific image positioning retrieval in random encrypted images in the past, such as the study which is based on the image's full-directional shape code to achieve the positioning of image features [3], in the process of positioning, the Omni-directional feature code of image shapes is constructed, the similarity between shapes is calculated, and the image positioning retrieval is realized according to the similarity. The method is simple and efficient, but the positioning accuracy is much lower; $\mathrm{Wu}$ et al. uses the grayscale symbiotic matrix to extract the 4 characteristic values of the detected image, introduces the weight factor with the direction measurement, fuses it with the 4 feature values, and forms the texture feature vector after Gaussian generalization processing, and uses the weighted average to fuse the feature distance of color and texture, but the feature accuracy of this method is low [4]; Huang et al. uses improved Henon map encryption to process the gray value of each band of the image, and obtains the feature vector based on the gray value to realize the effective positioning of the image [5]. This method has low computational complexity and low communication system overhead, but the frequency of acquiring image samples is low and takes a long time, so the requirement of random encrypted image real-time positioning cannot be achieved.

In view of the existing problems in the above literature, the method of multi-directional positioning retrieval of random encrypted images is proposed to realize the high-precision positioning retrieval of encrypted image features.

\section{The Multi-Directional Feature Positioning RETRIEVAL METHOD OF RANDOM ENCRYPTED IMAGES}

\section{A. Feature Extraction based on Multidirectional Binary} Wavelet Transform

Wavelet multi-resolution image decomposition algorithm

Multi-resolution thinking is widely used in computer vision and image processing, and multi-resolution decomposition image can reduce image size, the amount of image processing calculation, and also the degree of influence of a priori knowledge on image processing [6]. The core of the image decomposition algorithm based on wavelet multi-resolution 
analysis depends on the effective orthogonal wavelet base, which decomposes the specific image of the random image by two-dimensional wavelet [7].

Setting $g(x, y)$ shows specific image signal, $h$ represents the scale, discrete matrix 256256 is used to implement discretization, and the orthogonal basis of multidirectional binary wavelet is used to implement binary wavelet transform of dissociation in $h$, that is, the wavelet basis projection in three directions of a particular image, then there is:

$$
\begin{aligned}
W_{h, g_{1}, g_{2}}^{\prime} g=2^{h / 2} \quad{ } \quad g(u, v) F_{h, g_{1}, g_{2}}^{i} & \\
& \left(\begin{array}{lllll}
2{ }^{h} u & g_{1}, 2{ }^{h} v & g_{2}
\end{array}\right)
\end{aligned}
$$

In the formula, $W$ is picture content, $g$ is shift factor, $g(u, v)$ is picture signal, $F_{h, g_{1}, g_{2}}^{i}$ is orthogonal wavelet basis, $i=1,2,3$ is high-frequency component in three different directions, $0 \quad u, v<256$.

Referring to previous references, it can be obtained that through the two-dimensional wavelet projection transformation of (1), the components $\left(h_{1}, h_{2}, h_{3}\right)$ of the image signal in the horizontal, vertical and diagonal directions correspond to the high-frequency components in the three directions of the original image, so the low frequency region of the original image can be ignored. The above analysis process shows that the wavelet change itself has the function of dividing the high and low frequency components, and removing the low and keeping the high [8]. The high-frequency component of the image corresponds to the image edge information, that is, the components in the three directions of the image can be extracted by the multi-directional feature of the image.

\section{Image multi-directional feature extraction algorithm}

The main function of multi-resolution analysis in image edge retrieval is to reduce interference at low and high resolution, retrieve image edge and positioning accurately and quickly, obtain the actual orientation of image edge, and track the edge of the image from rough to fine, and determine the overall edge information of the image. Under the condition of $h$ fixed value, the feature information in the low-resolution image determines the edge of the high-resolution image. The three high-frequency components of the original low-resolution image that reflect the multi-directional characteristics of the image after the wavelet decomposition correspond to the three components in the horizontal, vertical and diagonal direction of the original image. Multidirectional binary wavelets have strong directivity. After the wavelet change processing, at local points in three directions, membranes $\left\|h_{1}\right\|,\left\|h_{2}\right\|,\left\|h_{3}\right\|$ of the three high-frequency components of the image are all extreme value. According to Canny algorithm, the edge of the image corresponds to the great value of the wavelet variation mode [9]. Thus, according to the local maximum value of the multidirectional binary wavelet transform membrane, the position and singularity of the signal mutation point are detected to extract the multidirectional feature of the image. In order to solve the problem of large calculation amount caused by extracting multi-directional features of the image in three directions through the threshold binarization method, the accuracy of the image edge is improved [10], the specific process is as follows:

1) Setting $R_{0}$ represents image edge segmentation threshold;

2) Through $R_{0}$ the maximum value of wavelet transform in the image is processed, the process is as follows:

If $\left(\|w\|>R_{0}\right) E_{i}=1 ; \quad E / s e E_{i}=0$

Of which, Eis edge point. Assume $\|w\|>R$, then set 1 as the grayscale value of this point, meanwhile, this point is considered as the edge point of the image; On the contrary, set 0 as the grayscale value of this point, then compute the next maximum point.

3) Superposition compute $E_{1}, E_{2}$ and $E_{3}$, and carry on the normalization processing;

$$
E_{0}={ }_{i=1}^{3} E_{i} /\left\|{ }_{i=1}^{3} E_{i}\right\|
$$

Of which, $E_{0}$ is final edge point. Connecting all $E_{0}$, the edge details of the image is obtained, and then multi-direction feature extraction of specific image in random encrypted image is realized.

\section{B. Optimization Method of Specific Image Positioning in Random Encrypted Image}

Based on the multi-directional features of specific images extracted from the previous section, in order to improve the accuracy of the positioning of specific images in random encrypted images, it is necessary to use image verification to exclude the excessive and repeated image features contained in specific images, and reduce the probability of image positioning errors [11] [12]. Although there may be more images similar to a particular image in positioning, due to the homogeneity of the discrete image to be classified, the impact of the same image in the positioning of a particular image can be reduced by iterative comparison of multiple identical images [13], the specific process is as follows:

$A_{h} B_{h} C_{h}$ is used to stand for the specific image verification areas, and $t$ is used to represent image feature point similar to multidirectional feature $\mathrm{E}$ of a particular image around $A_{h} B_{h} C_{h}$. Specific image feature points exist the only frame, equation (4) represents the weighted average value in its verification areal:

$\left(\begin{array}{ll}k & A\end{array}\right) \cdot m=0$ 
Of which, $m$ is the number of images, $k$ is a random directional feature vector for the unique frame feature of a particular image in the verification area, which needs to be achieved as follows in the verification area:

$k=k_{-}=\underline{x_{i}}+y_{i}+\underline{z_{h}}$

Of which, $x, y, z$ stands for horizontal, vertical and diagonal direction.

Assume the vector of $A$ in specific image verification area and the vector of image verification area are $A=x_{A} i+y_{A} j+Z_{A} h$ and $\underline{m}=a i+b j+c h$ respectively, then it can be certain that:

$$
a\left(\begin{array}{ll}
x & x_{A}
\end{array}\right)+b\left(\begin{array}{ll}
y & y_{A}
\end{array}\right)+c\left(\begin{array}{ll}
z & Z_{A}
\end{array}\right)=0
$$

According to the two-dimensional image features of the feature points $G$ and $W$ in the specific image positioning region, the linear equation of the specific image verification is determined as:

$$
\begin{aligned}
& \mathrm{r}=l i+o j+m h \\
& \mathrm{I}=x_{w} \quad x_{g}, o=y_{w} \quad y_{g}
\end{aligned}
$$

Of which, $r$ and represents a three dimensional first order equation of a plane.

Use association rules, the following formula can be obtained through (7):

$$
x=l e+x_{\mathrm{g}}, y=0 e+y_{g}
$$

Of which, $e$ is the wave coefficient and also the empirical coefficient of image features, and then equation (9) is used to get $e$ value:

$e_{T}=\frac{k}{s}$

In (9), $s$ is the similarity, $k=m g m A$, $s=a l+b o+c m$.

The new two-dimensional coordinates can be determined by (10):

$$
x_{q}=l e_{T}+x_{G}, y_{T}=o e_{T}+y_{G}
$$

During verification, the two-dimensional coordinate $(x, y)$ of a particular image within a randomly encrypted image need to be checked repeatedly to ensure the accuracy of the verification results.

The directivity of a particular image is not considered when determining the image with the same features as a particular image, determine the sub-graph $\left(\begin{array}{lll}1 & i\end{array}\right)$ with high degree of similarity in all identical images [14] [15].

All sub-graphs similar to a particular image are taken as candidate sets, and verify the candidate set to determine whether it is a specific image frequent item set [16].

Using vcode to stand vertex coding for each specific image frequent item set, $\{A, B, E\}$ is frequent item sets for specific images of the above example, its $\left[v 1, v 2, \cdots \in h_{n-1}\right]$ and $c\{v 1, v 2, \cdots\} \mid v 1, v 2, \cdots \in h_{n}$ are the sets of frequent item sets and frequent candidate sets of specific images respectively.

With a specific image frequent item set, set the unique specific image feature as $A_{h} B_{h} C_{h}$, the problem of specific images positioning influenced by excessive and duplicate image is seen as whether there is a problem within the verification area [17]. Check whether all feature points in the region are coincide with the same feature points $T$, determine whether the current image is disturbed by other images, if there is a feature point in the verification area overlaps with $T$, then the current image is a specific image [18] [19].

\section{EXPERIMENTAL ANALYSES}

\section{A. Detection of Encrypted Sample Image by Different} Methods

Comparing the method of this paper, the random encrypted image positioning retrieval method with all-directional shape feature code and the positioning retrieval performance of the random encrypted image location retrieval method based on the improved color histogram and gray scale symbiotic matrix in experiments, the results are as follows.

The randomly encrypted sample image in the image is shown in Fig. 1, and different methods are used to locate the encrypted sample image, the result is shown in Fig. 2.

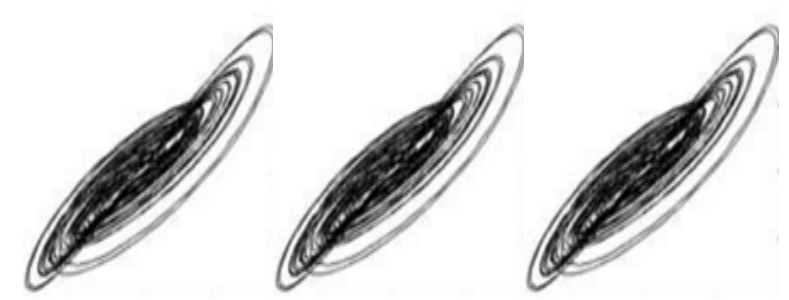

Fig. 1 the randomly encrypted sample image

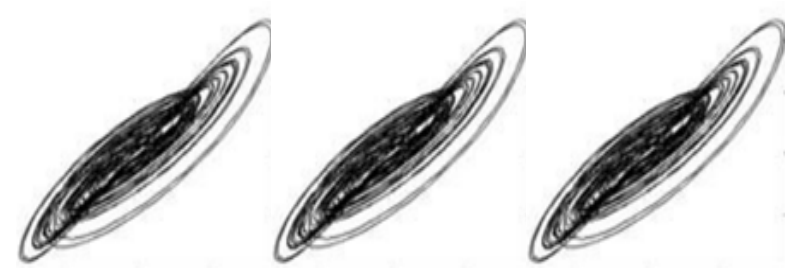

(a). the positioning retrieval result of the method in this paper 


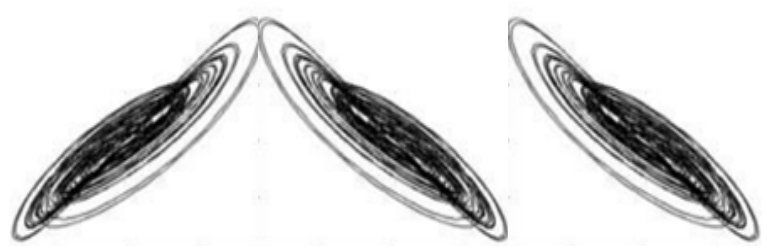

(b). positioning retrieval result with all-directional shape feature code
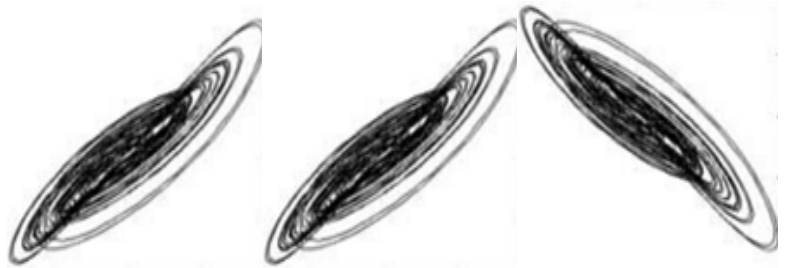

(c) the positioning retrieval result of the improved color histogram and gray scale symbiotic matrix method

Fig. 2 the comparing results of different methods of experiments

Fig. 2 is a visual representation, and the image positioning retrieval results obtained by the method in this paper are consistent with the image features of random encryption samples; The positioning retrieval results of the all-directional shape feature code retrieval method have some horizontal opposite states with that of random encrypted sample images, and some of the positioning retrieval results of the color histogram and grayscale symbiotic matrix methods are in vertical opposite state with that of random encrypted sample images.

B. Comparison Test of Result Accuracy of Different Positioning Methods

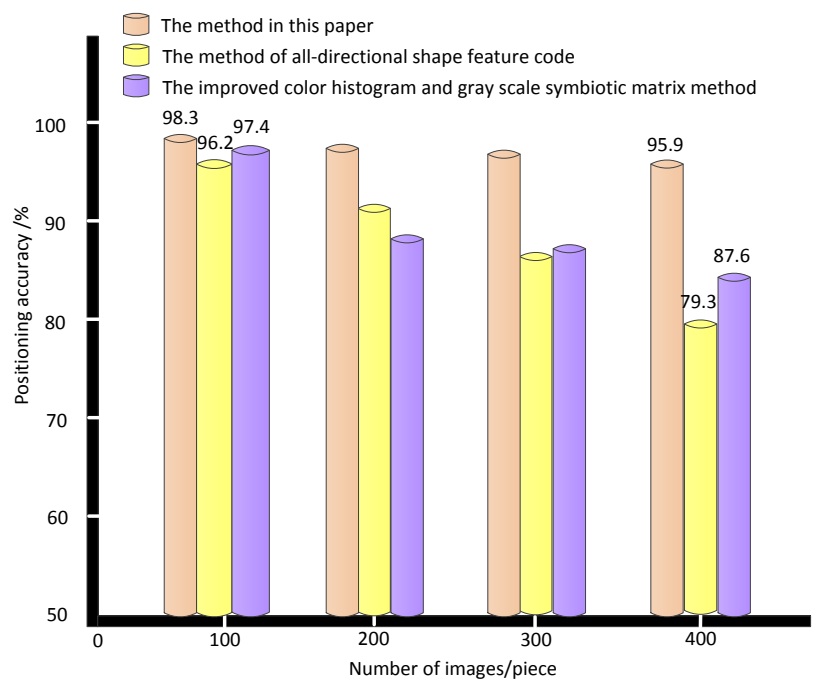

Fig. 3 the result accuracy trend chart of different positioning methods

As shown in Fig. 3, when the number of images is 100, the difference of image feature location accuracy between the three methods is small. With the increase of the number of images, the positioning accuracy of the three methods is gradually declining. The positioning accuracy of the omni-directional shape feature code method is reduced to $90 \%$, and the positioning accuracy of the improved color histogram and gray common incidence matrix method is lower than $90 \%$. However, the downward trend of this method is relatively slow, which is almost the same as that of 100 images. With the increase of the number of images, the positioning accuracy gap of the three methods increases gradually. When the number of images reaches 400 , the positioning accuracy of the improved color histogram and gray common incidence matrix method is reduced to about $83 \%$, and the positioning accuracy of the omni-directional shape feature coding method has been reduced to less than $80 \%$, while the positioning accuracy of this method remains above $95 \%$, which is always higher than that of the contrast method, and is very stable. The main reason is that the method has the function of feature verification, which can significantly improve the accuracy of data correlation and greatly improve the accuracy of image feature location and retrieval.

\section{Accuracy Ratio Test of Image Feature Points Based on} Different Location Retrieval Method

Comparing the precision ratio of image feature points of three different positioning retrieval methods in the same experimental environment, the results are shown in Table I.

Table. I Comparison results of precision ratio of image feature points extracted by different methods $/ \%$

\begin{tabular}{|c|c|c|c|}
\hline $\begin{array}{c}\text { Image } \\
\text { sequence } \\
\text { number }\end{array}$ & $\begin{array}{c}\text { Method in } \\
\text { this paper } \\
(\%)\end{array}$ & $\begin{array}{c}\text { All - } \\
\text { directional } \\
\text { shape } \\
\text { feature } \\
\text { code } \\
\text { method } \\
(\%)\end{array}$ & $\begin{array}{l}\text { The methods of } \\
\text { improved color } \\
\text { histogram and gray } \\
\text { co-occurrence } \\
\text { matrix }(\%)\end{array}$ \\
\hline 1 & 97.3 & 92.2 & 94.3 \\
\hline 2 & 96.8 & 91.5 & 97.2 \\
\hline 3 & 98.4 & 93.6 & 94.8 \\
\hline 4 & 96.9 & 90.7 & 93.1 \\
\hline 5 & 95.6 & 92.8 & 93.9 \\
\hline 6 & 97.7 & 92.2 & 95.2 \\
\hline 7 & 98.0 & 91.7 & 96.4 \\
\hline 8 & 98.2 & 92.5 & 95.5 \\
\hline 9 & 97.7 & 94.0 & 93.1 \\
\hline 10 & 97.6 & 94.8 & 94.6 \\
\hline 11 & 96.1 & 94.2 & 96.5 \\
\hline 12 & 95.2 & 94.0 & 96.3 \\
\hline 13 & 98.6 & 91.5 & 95.0 \\
\hline 14 & 96.3 & 92.8 & 97.0 \\
\hline 15 & 98.6 & 94.2 & 93.3 \\
\hline
\end{tabular}

In order to more clearly reflect the difference in the precision ratio of feature points in the feature extraction process of three different positioning retrieval methods, the data in Table I is described in the form of a line chart, as shown in Fig. 4. 


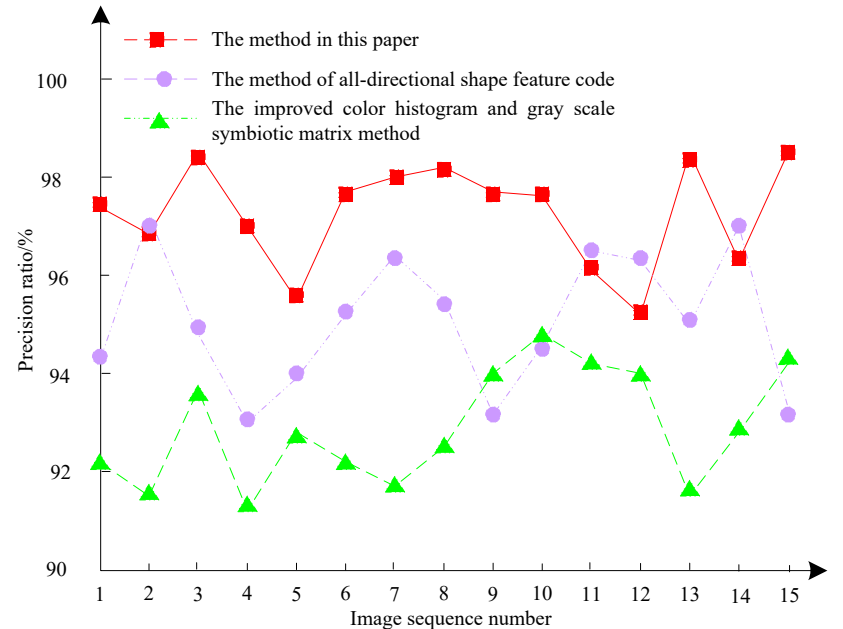

Fig. 4 the contrast results of the feature extraction process of different methods

According to Table I and Fig. 4, the accuracy ratio of feature points fluctuates from $90.7 \%$ to $94.8 \%$, and the average accuracy ratio is about $92.75 \%$; The accuracy ratio of the improved color histogram and gray common incidence matrix method fluctuates from $93.1 \%$ to $97.2 \%$, and the average accuracy ratio is about $95.15 \%$; However, the accuracy ratio of feature points fluctuates from $95.2 \%$ to $98.6 \%$, and the average accuracy ratio is about $97.3 \%$, which is $4.55 \%$ higher than the omnidirectional shape feature code method, and $2.15 \%$ higher than the improved color histogram and gray common incidence matrix method. The results show that this method has high accuracy for randomly encrypted images in the process of feature extraction. The results show that this method has high accuracy in the process of feature extraction.

In order to verify the anti-noise performance of the proposed method in this paper, the peak signal to noise ratio and structural similarity of different methods are compared under the conditions of Gaussian noise interference and salt and pepper noise interference, respectively. The results are shown in Fig. 5 and Fig. 6.

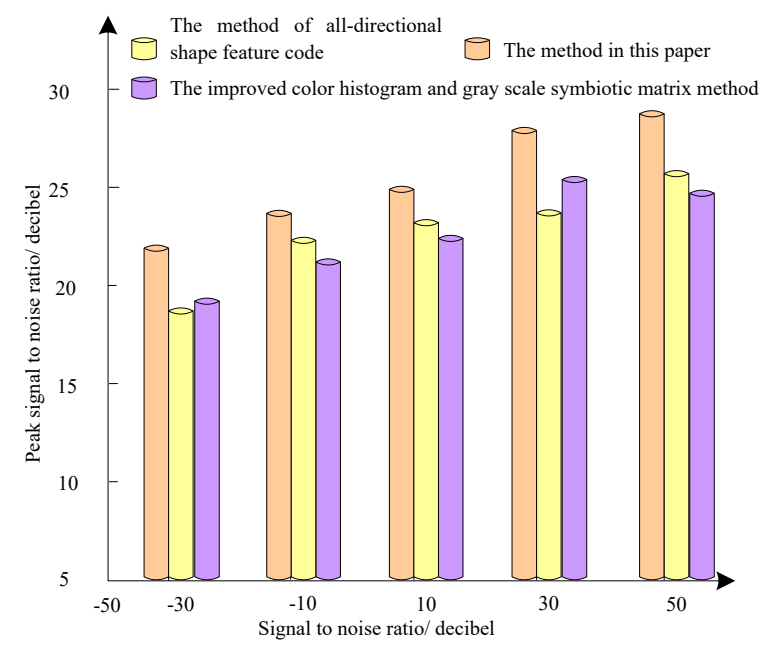

(a). peak signal to noise ratio at different signal to noise ratios

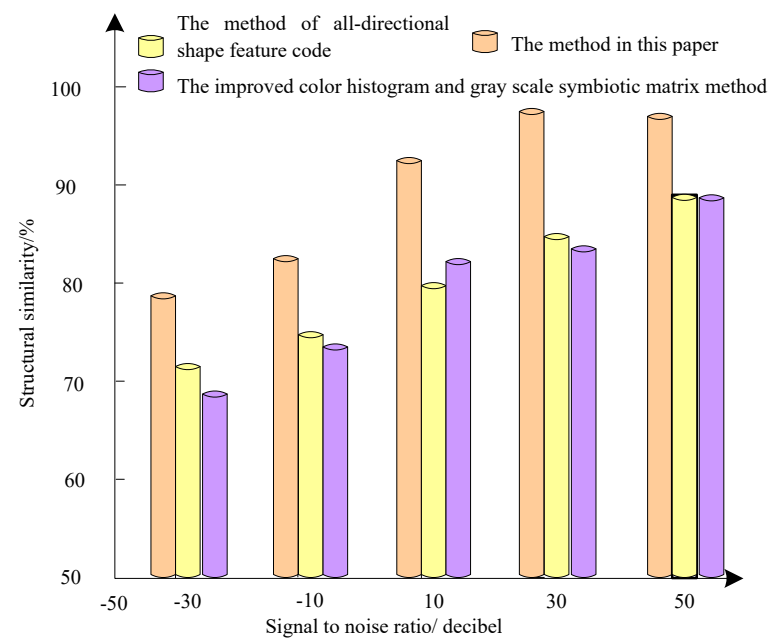

(b). structural similarity values under different signal to noise ratios Fig. 5 Comparison of anti-noise performance of different methods under Gaussian noise interference

According to Fig. 5, under the interference of Gaussian noise, with the improvement of the signal to noise ratio, the peak signal to noise ratio and the structural similarity of the three methods all show varying degrees of upward trend. In contrast, the increase in the method of this paper is obvious higher than that of the other two methods. It shows that the proposed method is superior to the other two methods in the anti-noise performance of Gaussian noise.

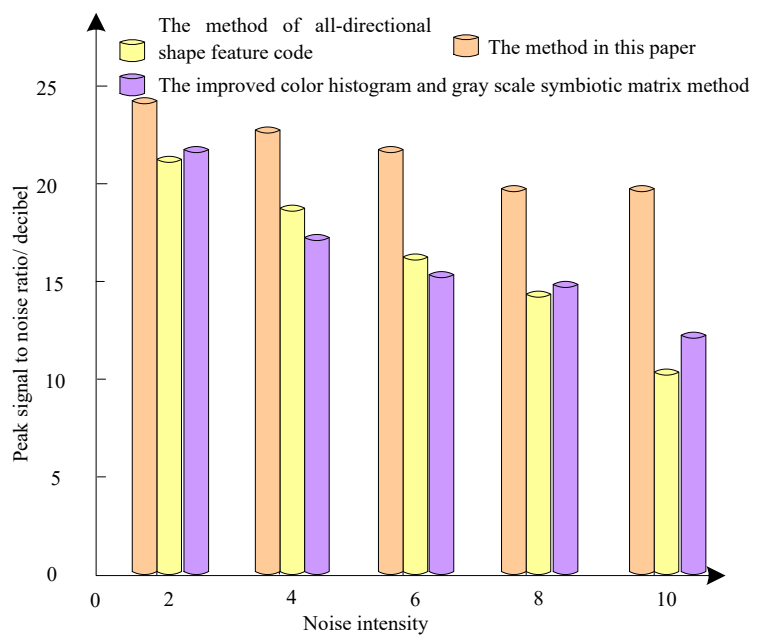

(a). the influence of noise intensity on peak signal to noise ratio 


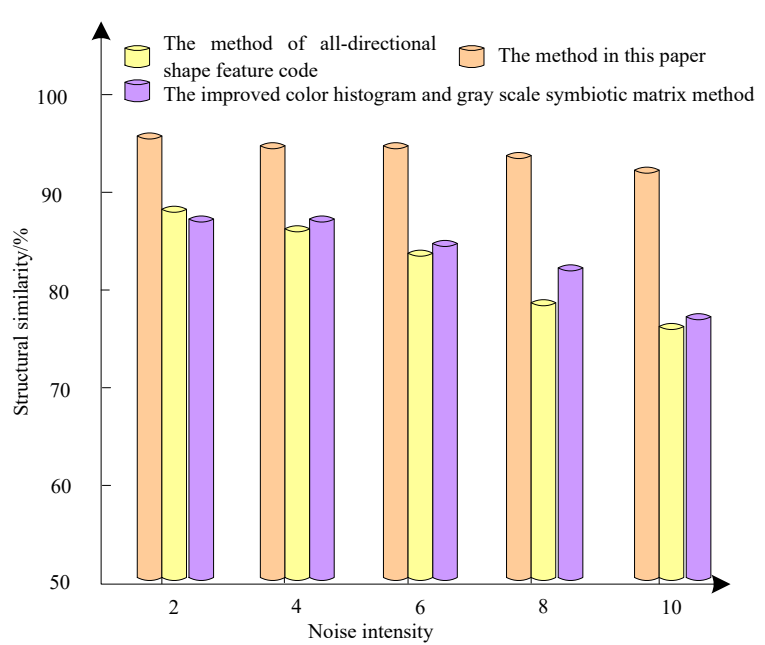

(b). The influence of noise intensity on structural similarity

Fig. 6 comparison of anti-noise performance of different methods under the interference of salt and pepper noise

It can be seen from Fig. 6 that under the interference of pepper noise and salt noise, with the increase of noise intensity, the peak signal-to-noise ratio and structural similarity of the three methods show a downward trend in varying degrees.

The peak signal-to-noise ratio of omnidirectional shape feature code method is reduced from 22 to 10 , the improved color histogram and gray common incidence matrix method is reduced from 23 to 12 , and the proposed method is reduced from 24 to 20. Compared with the other two methods, the performance degradation of this method is slower, which shows that this method has better antinoise performance than the other two methods.

The structural similarity of all-round shape feature code method, improved color histogram and gray common incidence matrix method is reduced from 88 to 76 , which is always lower than 90 . Although the similarity structure of this method is also in a state of continuous decline, from 96 to 94 , the downward trend is relatively slow, which indicates that the similarity structure of this method is better.

From the comprehensive analysis of the experimental results in Fig. 5 and Fig. 6, it can be concluded that compared with the other two methods, the method in this paper has a higher anti-noise performance.

\section{CONCLUSION}

The encryption of images can effectively improve the security of image information content. In view of the low positioning accuracy and feature precision ratio problems in the previous random encrypted image positioning retrieval method, this paper studies the multi-directional feature positioning retrieval method of random encrypted image, extracts the multi-directional features of specific images in random encrypted images by using multidirectional binary wavelet transform method, and locates and retrieves the random encrypted specific images according to the frequent set that are identical to a particular image feature in the random encrypted image.

The results show that the positioning accuracy of this method is more than $95 \%$, the average accuracy ratio of feature points is $97.3 \%$, the peak signal-to-noise ratio and structural similarity show a slow downward trend, and it has better antinoise performance, and the practical application effect is better.

\section{ACKNOWLEDGMENT}

General program of Hainan Natural Science Foundation (No. 617016); Research project of education and teaching reform of Hainan University (No. hdjy2054).

\section{Reference}

[1] H. L. Guo, L. Liao, and J. Yang, "Simulation of efficiently specific image positioning method in random encryption image," Computer Simulation, vol. 32, no. 3, pp. 434-437, 2015.

[2] X. Zhao, Y.Y. Xu, J.Y. Gong, and F. Z. Song, "A secure image retrieval method based on combined orthogonal decomposition and BoVW," Journal of Applied Sciences, vol. 36, no. 2, 299-308, 2018.

[3] Z. Y. Tian, C. G. Men, Y. N. Tang, and Q. F. Jiang, "Image retrieval based on the omnidirectional shape feature code," Journal of Harbin Institute of Technology, vol. 48, no. 11, 129-134, 2016.

[4] Q. T. Wu, Z. H. Cao, and J. F. Shi, "Image retrieval based on improved color histogram and gray level co-occurrence matrix," Journal of Graphics, vol. 38, no. 4, pp. 543-548, 2017.

[5] D. M. Huang, X. Geng, L. F. Wei, and S. U. Cheng, "A secure query scheme on encrypted remote sensing images based on Henon mapping," Journal of Software, vol. 27, no. 7, pp. 1729-1740, 2016.

[6] Y. Li, Z. W. Wang, S. F. Xu, and Y. L. Zhang, "Image encryption algorithm based on multiple-parameter fractional Fourier and Arnold transforms," Journal of China Academy of Electronics and Information Technology, vol. 11, no. 2, pp. 164-168, 2016.

[7] K. Muhammad, M. Sajjad, I. Mehmood, S. Rho, and S. W. Baik, "A novel magic LSB substitution method (M-LSB-SM) using multi-level encryption and achromatic component of an image," Multimedia Tools \& Applications, vol. 75, no. 22, pp. 1-27, 2016.

[8] L. Xie and Y. Cheng, "Image retrieval based on color and motif gradient direction co-occurrence histogram," Computer Engineering and Applications, vol. 52, no. 10, pp. 181-186, 2016.

[9] Y. C. Fan, X. H. Tan, M. Q. Zhou, and X. Zheng, "3D model sketch retrieval method based on local multi-scale," Chinese Journal of Computers, vol. 40, no. 11, pp. 18-35, 2017.

[10]R. B. Tu, Z. H. Chen, and H. K. Wang, "One joints localization in mouse micro-CT images using random forests algorithm," Chinese Journal of Biomedical Engineering, vol. 36, no. 3, pp. 257-266, 2017.

[11] S. K. Vipparthi, S. Murala, and S. K. Nagar, "Dual directional multi-motif XOR patterns: A new feature 
descriptor for image indexing and retrieval," Optik-International Journal for Light and Electron Optics, vol. 126, no. 15-16, pp. 1467-1473, 2015.

[12] G. Song, X. Jin, and X. Y. Tan, "Near-duplicate image retrieval based on multi-features bundle," Pattern Recognition and Artificial Intelligence, vol. 29, no. 10, pp. 943-950, 2016.

[13] M. C. Xu and X. H. Wang, "The study of texture features extraction based on incomplete tree-structured wavelet transform," Journal of Image and Graphics, vol. 14, no. 7, pp. 1341-1346, 2018.

[14] M. Zhao, H. X. Zhang, and L. Liu, "Image retrieval method based on color and edge orientation," Journal of Data Acquisition \& Processing, vol. 31, no. 3, pp. 577-583, 2016.

[15] M. Zhao, H. Zhang, and J. Sun, "A novel image retrieval method based on multi-trend structure descriptor," Journal of Visual Communication \& Image Representation, vol. 38, no. C, pp. 73-81, 2016.

[16] S. A. Stankevich, M. A. Popov, S. V. Shklyar, K. Y. Sukhanov, and B. Sun, "Subpixel-shifted satellite images superresolution: software implementation," WSEAS Transactions on Computers, vol. 19, no. 5, pp. 31-37, 2020.

[17] R. Mamdouh, H. M. El-Bakry, A. Riad, and N. El-Khamisy, "Converting 2D-medical image files "DICOM" into 3Dmodels, based on image processing, and analysing their results with Python programming," WSEAS Transactions on Computers, vol. 19, no. 2, pp. 10-20, 2020.

[18]F. E. Ekpar, H. Hase, and M. Yoneda, "Visualizing very large image data sets at interactive rates," WSEAS Transactions on Signal Processing, vol. 16, no. 11, pp. 89-98, 2020.

[19] V. Ponomaryov and A. Palacios-Enriquez, "Visualizing very large image data sets at interactive rates," WSEAS
Transactions on Signal Processing, vol. 16, no. 11, pp. 89-98, 2020.

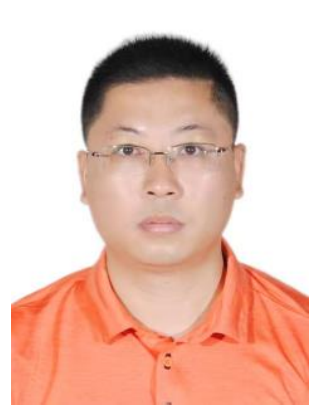

Fuwei Huang, male, born in 1980 in Lingao, Hainan, lecturer, master, graduated from the computer application major of Hainan University, now teaches in the school of computer science and technology of Hainan University. His research fields are blockchain and Internet of things applications. He has published 8 papers in core journals and national journals, presided over 1 provincial scientific research project and 1 university scientific research project, Participated in 3 provincial projects and 2 textbook compilation.

\section{Contribution description}

Fuwei Huang passed the experiment, the multi-direction feature retrieval method of random encrypted images is studied comprehensively. Multidirectional binary wavelet is used to decompose specific images, and multi-resolution analysis is used to extract multi-direction features of specific images. The results show that the method can locate the random encrypted image effectively.

\section{Creative Commons Attribution License 4.0 (Attribution 4.0 International, CC BY 4.0)}

This article is published under the terms of the Creative Commons Attribution License 4.0 https://creativecommons.org/licenses/by/4.0/deed.en_US 